\title{
Apocrine Sweat Gland Hamartoma
}

National Cancer Institute

\section{Source}

National Cancer Institute. Apocrine Sweat Gland Hamartoma. NCI Thesaurus. Code C5563.

A hamartoma characterized by localized apocrine sweat gland malformation. 